\title{
Tuberculous pyomyositis of the temporal muscle in a non- immunocompromised woman: diagnosis by sonography
}

\author{
Chin-Kai Chu, M.D., Tsung-Lin Yang, M.D.*, Ching-Ting Tan, M.D., Ph.D.*
}

\begin{abstract}
Pyomyositis (PM) is an uncommon cause of acute bacterial infection occurring in skeletal muscle. This disease is rare in non-tropical areas, and PM caused by Mycobacterium is very rare in a non-immunocompromised person. The presence of temporal area swelling may lead to a differential diagnosis toward more common pathologies, such as a complication of mastoiditis or a neoplasm of the temporal area. This article describes a case of tuberculous pyomyositis in a non-immunocompromised woman, the diagnosis of which was confirmed by sonographically guided fine-needle aspiration.
\end{abstract}

Key words: Sonography, Sound; Tomography, X-Ray Computed; Temporal Muscle; Tuberculosis; Biopsy, Needle

\section{Introduction}

Pyomyositis (PM) is a bacterial infection occurring in skeletal muscle with no obvious local or adjacent source of infection. The entity is rare in non-tropical areas, and the most commonly isolated pathogen is Staphylococcus aureus. PM caused by Mycobacterium sp. is very unusual and has rarely been reported in a non-immunocompromised person. This is why the diagnosis of tuberculous PM is often delayed. This article describes the first case of tuberculous PM of the temporal muscle in a 29-year-old non-immunocompromised Taiwanese woman diagnosed on the basis of sonographic findings and sonographically guided fine-needle aspiration.

\section{Case report}

A 29-year-old woman was referred as an emergency with a two week history of left temporal area swelling and tenderness. She had suffered from left otalgia with intermittent otorrhoea for six months. In addition, progressive left-sided headache with mild fever had been noted in the most recent three weeks. She denied a history of diabetes mellitus, intravenous drug addiction or other major systemic diseases. She reported no recent trauma, insect bites, skin infections, or any travel to tropical countries.

On physical examination, marked swelling and extreme tenderness to light touch were noted in the left temporal area, but no erythema was detected over that area. There was noticeable trismus. Ear inspection revealed a normal appearance of the eardrum, with a little discharge from the roof of the left external ear canal. The remainder of the physical examination was unremarkable. Laboratory results revealed an elevated white blood cell count at $13.1 \mathrm{k}$ without a marked left shift. Tests for HIV were negative. Sonography was performed and revealed an enlarged echogenic temporal muscle with a hypoechoic lesion on the left temporal area (Figure 1A) compared

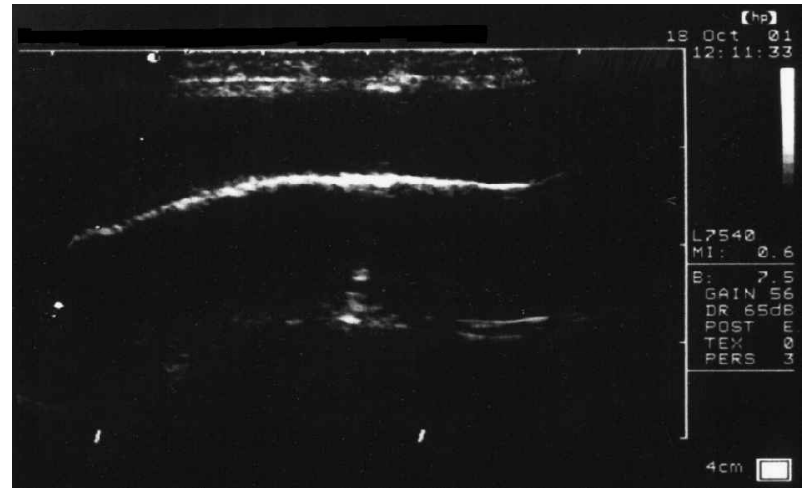

(a)

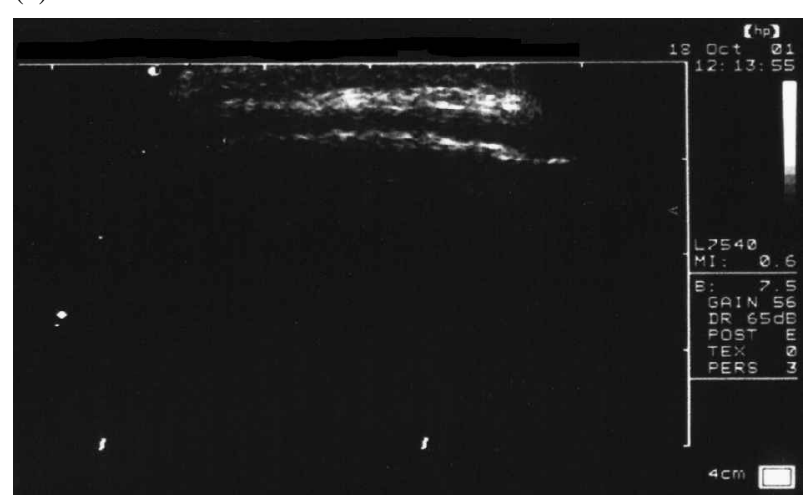

(b)

\section{FIG. 1}

A. Transverse sonogram of the swollen left temporal area showing a hypoechoic mass with slightly increased throughtransmission. The enlarged temporal muscle has become echogenic and the subcutaneous tissue has also increased. Sonographically guided aspiration yielded thick, purulent material. B. Sonogram of the normal right-side temporal area.

From the Departments of Otolaryngology, Lo-Tung Poh-Ai Hospital, Ilan 265, Taiwan and the National Taiwan University Hospital and National Taiwan University College of Medicine*, Taipei 100, Taiwan.

Accepted for publication: 23 September 2003. 
- This is a report of pyomyositis due to tuberculosis in the temporal muscle

- The patient was not immunocompromised and the diagnosis is seldom made, especially in subjects who are immunocompetent

- The diagnosis was confirmed by ultrasound-guided fine-needle aspiration biopsy and CT scanning used to elucidate the extent of the disease

- This differential diagnosis is discussed

with a normal right temporal area (Figure 1B). Under suspicion of temporal muscle swelling with abscess formation, echo-guided fine-needle aspiration of the swollen region was performed. About $1.5 \mathrm{ml}$ of whitish pus was obtained, and a diagnosis of PM was made. Computed tomography (CT) scan was arranged to evaluate the extent of the disease and the condition of the temporal bone. This revealed marked swelling of the left temporal muscle without definite mastoiditis (Figure 2). Intravenous amoxicillin and clavulanate potassium were prescribed for treatment of the infection. Routine bacterial (aerobic and anaerobic) and fungal cultures of the pus yielded no growth. However, the microscopic examination showed numerous acid-fast bacilli ( $>5$ per oil immersion field), and an assay based on polymerase chain reaction showed a positive result for Mycobacterium tuberculosis. Chest X-ray revealed no nodules or other possible abnormalities caused by Mycobacterium tuberculosis. The treatment was changed to antituberculosis agents, including isoniazid, rifampicin, pyrazinamide and ethambutol. After one month of treatment the swelling and tenderness of the left temporal area had significantly decreased, and repeat sonography revealed reduced soft tissue swelling with no abscess formation. After 12 months of treatment the patient was well with no discomfort and so the medication was discontinued.

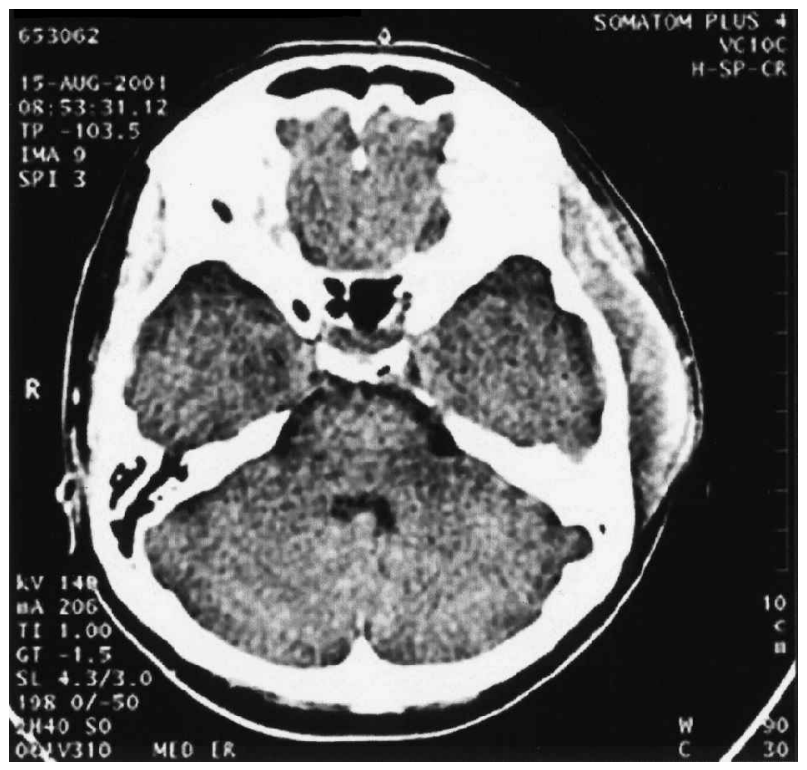

FIG. 2

CT scan revealing marked swelling of the left temporal muscle without definitely acute inflammatory changes of the underlying bone.

\section{Discussion}

PM is a rare pyogenic bacterial infection of a large skeletal muscle, usually followed by abscess formation. ${ }^{1}$ Originally, most cases were reported in tropical regions. ${ }^{2} \mathrm{PM}$ patients frequently have a recent history of trauma, and some of them may have a history of recent or concurrent skin or respiratory infections. ${ }^{3}$ The most common aetiologic agent is Staphylococcus aureus, which is responsible for about 85 per cent of cases in both tropical and non-tropical zones. ${ }^{4}$ Tuberculosis of the striated muscle is rare even in countries where tuberculosis is a relatively common disease. ${ }^{5}$ Normally, skeletal muscle is highly resistant to bacterial infection and abscess formation. ${ }^{6}$ Most cases from temperate regions involve patients with an underlying immunocompromised condition such as diabetes mellitus, ${ }^{7}$ longterm corticosteroid treatment, ${ }^{8}$ postorgan transplantation ${ }^{9}$ or infection with HIV. ${ }^{10,11}$ PM predominantly involves a single large muscle such as the muscles of the thigh, back, buttock or arm. ${ }^{3}$ To our knowledge, this is the first case report of tuberculous PM involving the temporal muscle in a healthy young person.

$\mathrm{PM}$ is difficult to diagnose clinically because of many other pathological processes (including osteomyelitis, fasciitis, cellulitis, abscess formation, polymyositis, haematoma, etc.) that may produce symptoms similar to it. ${ }^{12} \mathrm{CT}$, magnetic resonance imaging (MRI) and sonography have all been reported as diagnostic tools for PM. CT is of value for localization of the lesion and to differentiate it from a haematoma, cellulites or deep venous thrombosis. ${ }^{13}$ It can also be useful in guiding a percutaneous biopsy and/or partial drainage procedures. ${ }^{14}$ MRI with gadolinium injection was found to be not only useful in helping to make the diagnosis, but also sensitive in detecting coexisting bone changes such as osteomyelitis. ${ }^{15,16} \mathrm{CT}$ and MRI are valuable tools in the assessment of PM and in determining its location and extension. However, they are expensive and not always accessible in emergency rooms or outpatient clinics. Ultrasound is a readily available and definitive examination for PM. ${ }^{17}$ Although sonography was found to underestimate the extent of the disease, it can effectively detect fluid collections. ${ }^{14}$ It can thus be used to search for the optimal aspiration or biopsy site. ${ }^{18}$ In addition, repeat sonography is useful for following up the disease condition. ${ }^{3}$ In the case described here, ultrasound not only played a critical role in the early diagnosis of PM, which allowed us to avoid surgical exploration, but also provided valuable evaluation of the treatment results.

Because of its rarity and deceptive clinical presentation, PM may go unrecognized for weeks in non-tropical regions and may cause severe morbidity and mortality. Failure to recognize this clinical entity can lead to diagnostic delay and inappropriate management. PM should be included in the differential diagnosis of patients with unexplained muscle pain and swelling, even in non-immunocompromised patients. In addition, sonography is a convenient, economical, non-invasive and readily available instrument for the early diagnosis of PM.

\section{References}

1 Gibson RK, Rosenthal SJ, Lukert BP. Pyomyositis. Increasing recognition in tempertae climates. Am J Med 1984;77:768-72

2 Levin MJ, Gardner P, Waldvogel FA. Tropical pyomyositis. N Engl J Med 1971;284:196-8

3 Quillin SP, McAlister WH. Rapidly progressive pyomyositis. Diagnosis by repeat sonography. J Ultrasound Med 1991;10:181-4

4 Chiedozi LC. Pyomyositis: review of 205 cases in 112 patients. Am J Surg 1979;137:255-9 
5 Bonomo RA, Graham R, Makley JT, Petersilge CA. Tuberculous pyomyositis: an unusual presentation of disseminated Mycobacterium tuberculosis infection. Clin Infect Dis 1995;20:1576-7

6 Brown JD, Wheeler B. Pyomyositis: report of 18 cases in Hawaii. Arch Intern Med 1984;144:1749-51

7 Wang TK, Wong SS, Woo PC. Two cases of pyomyositis caused by Klebsiella pneumoniae and review of the literature. Eur J Clin Microbiol 2001;20:576-80

8 Kim HA, Yoo CD, Baek HJ, Lee EB, Ahn C, Han JS, et al. Myocobacterium tuberculosis infection in a corticosteroid-treated rheumatic disease patient population. Clin Exp Rheumatol 1998;16:9-13

9 Johnson DW, Herzig KA. Isolated tuberculous pyomyositis in a renal transplant patient. Nephrol Dial Transplant 2000;15:743

10 Gaut P, Wong PK, Meyer RD. Pyomyositis in a patient with the acquired immunodeficiency syndrome. Arch Intern Med 1988;148:1608-10

11 Antony SJ, Kernodle DS. Nontropical pyomyositis in patients with AIDS. J Natl Med Assoc 1996;88:565-9

12 Moore DL, Delage G, Labelle H, Gauthier M. Peracute streptococccal pyomyositis: report of two cases and review of the literature. J Pediatr Orthop 1986;6:232-5

13 McLoughlin MJ. CT and percutaneous fine-needle aspiration biopsy in tropical myositis. Am J Roentgenol 1980;134:167-8

14 Sharif HS, Clark DC, Aabed MY, Aideyan OA, Haddad MC, Mattsson TA. MR imaging of thoracic and abdominal wall infections: comparison with other imaging procedures. Am J Roentgenol 1990;154:989-95
15 Kim JY, Park YH, Choi KH, Park SH, Lee HY. MRI of tuberculous pyomyositis. $J$ Comput Assist Tomogr 1999;23:454-7

16 Spiegel DA, Meyer JS, Dormans JP, Flynn JM, Drummond DS. Pyomyositis in children and adolescents: report of 12 cases and review of the literature. J Pediatr Orthop 1999;19:143-50

17 Royston DD, Cremin BJ. The ultrasonic evaluation of psoas abscess (tropical pyomyositis) in children. Pediatr Radiol 1994;24:481-3

18 Gottlieb RH, Meyers SP, Hall C, Amesur N, Domke R, Rubens DJ. Pyomyositis: diagnostic value of color Doppler sonography. Pediatr Radiol 1995;25(Suppl 1):S109-11

Address for correspondence:

Ching-Ting Tan, M.D., Ph.D.,

Department of Otolaryngology,

National Taiwan University Hospital and National Taiwan

University College of Medicine,

Taipei 100, Taiwan.

Fax: 886-2-23410905

E-mail: christin@ha.mc.ntu.edu.tw

Dr C. T. Tan takes responsibility for the integrity of the content of the paper.

Competing interests: None declared 